\title{
Thé au harem d'Archimède: un peu de couleur dans la grisaille
}

\author{
Danielle Marx-Scouras \\ The Ohio State University
}

L e spectateur du Thé au harem d'Archimède 'sombre tout de suite dans la noirceur du film où il décèle à peine deux spectres perdus dans l'obscurité de la nuit. C'est le moment précaire où le prolétaire de la banlieue parisienne entame ce long parcours qui l'emmenera des HLM de la cité défavorisée à l'autre galère, la chaîne de montage à l'usine. L'on visite rarement les lieux de travail dans le film comme dans le roman. L'obscurité de la nuit nous en dit plus. Bien avant l'aube, la prolétaire Josette emmène son fils Stéphane chez la voisine avant de commencer le long chemin du travail à l'autre bout de Paris. Le roman reprend ce même moment: "Le jour se lève à peine sur la banlieue ringarde, la nuit fait la grasse matinée" (43). A travers cette personnification plutôt ironique, Charef exprime les désirs que ses personnages n'osent plus avoir. En effet, la nuit n'a rien de rassurante.

Petit à petit, nous passons de l'obscurité de la nuit à la grisaille du petit matin parisien. La grisaille du moment est renforcée par celle du lieu: la caméra accentue les HLM de béton gigantesques, à côté desquels les deux personnages semblent perdus. L'environnement fait sentir son poids sur ses habitants. La musique de la chanson "Banlieue" de Karim Kacel se prononce à peine comme un air langoureux.

Cette première scène contraste dramatiquement avec celle de la traversée du seuil de l'appartement de Malika où Josette dépose son fils avant de partir au travail. L'accueil chaleureux des Français par cette famille algérienne est renforcée par la lumière qui pénètre le film à ce moment. Le petit Stéphane est pris en charge par Malika et sa famille, avec qui il partagera le petit déjeuner avant d'être accompagné à l'école par un des enfants. Cette première rencontre entre familles défavorisées venant de deux cultures différentes est fort positive: ici, l'identité n'est ni française, ni algérienne; elle est plutôt d'ordre économique - on s'entre-aide comme on peut. Dans la scène suivante, le jour se lève sur une usine occupée: mais, en peu de jours, Josette ne sera plus gréviste mais chômeuse. Les premières minutes du film nous mettent dans le bain. Un bain qui est ausi celui d'Archimède: "Tout corps plongé dans un liquide subit une poussée verticale, dirigée de bas en haut, égale au poids du fluide déplacé et appliqué au centre de gravité de ce fluide." 
A travers son titre ironique, Charef ferait-il aussi allusion à ces corps immigrés, étrangers qui déplacent et modifient le centre de gravité de la société française?

Dans le roman où la couleur grise représente le béton et symbolise la misère des habitants de la Zone, où l'on fuit la misère par la promiscuité sexuelle, l'alcool, et la drogue, Charef introduit cependant une autre couleur - le vert bouteille. Elle apparaît, la première fois dans le roman, pour décrire le salon de Malika: "Il n'y a pas de fauteuil dans ce salon, ni de plantes, rien qui fasse luxe ou décoration. Seulement un lit contre le mur, bien recouvert d'un couvre-lit vert bouteille que chacun des enfants veut s'approprier pour regarder la télevision" (18). Dans ce salon austère, où la télévision reste la seule forme d'évasion, le vert bouteille est une couleur factice qui connote l'absence. Ce vert manufacturé par les chaînes de montage attire plutôt l'attention sur le manque de verdure, de même que ces noms d'allées dans la cité: allée des Acacias, allée des Azalées — "La Cité des Fleurs, que ca s'appelle!!!" (22). Comme ces noms de rues, le vert bouteille est un porte-à-faux. Il s'agit d'une ironisation tout à fait plausible de la part d'un écrivain et cinéaste qui avait travaillé pendant des années dans une usine en tant qu'affûteur. Charef fabriquait des outils pour les jardins des bourgeois! Les fruits de son labeur étaient récoltés ailleurs. Le vert bouteille renforce ce qui existe ailleurs mais manque ici, c'est-àdire les loisirs d'autrui rendus possibles par le travail ingrat des défavorisés. Sur les chemins de la Zone, on trouve plus facilement des bouteilles abandonnées ou cassées que des plantes et des fleurs: "Les fleurs! les fleurs!...Et sur les murs de béton, des graffiti, des slogans, des appels de détresse, des S.O.S. en forme de poing levé" (22) Comme le nom de ces allées où la nature ne montre guère son visage, ce vert bouteille manufacturé représente les quelques minutes de pause offertes aux travailleurs épuisés par leurs journées interminables à la chaîne: qu'il s'agit de la bouteille de bière ou de coca cola qui fixe "l'heure de la mort à l'ouvrage/et de la pause qui rafraîchit/et ravigote le dollar.""

Le vert bouteille est aussi la couleur de prédilection pour les cravates des Gitans marginalisés dans leur campement sur les terrains vagues (69). Mais leurs femmes, que Magid admire et désire tant, "sont toujours en technicolor...Elles portent des robes longues aux teintes criardes, d'un jaune vif et aveuglant, presque phosphorescent, décorées de roses, un rose outrageant, clinquant, comme un coup sur la tête" (68). Aussi criardes et artificielles que ces couleurs puissent sembler, elles n'empêchent pas les personnages de rêver à une réussite colorée d'une autre manière: "Quand Majid les observe, il se demande toujours ce que de belles femmes comme elles fichent accrochées à une caravane, une gamelle dégueulasse à la main, dans ces sordides trous de terrain vague. Lui, il les emmènerait, ces belles dames, vers une pâtisserie huppée et leur ferait un lit entre les dragées de toutes les couleurs, les blanches rondes, les bleues ovales et les roses plates. Comme quand on était gosse, léchant la vitrine" (68). Les couleurs des riches sont beaucoup plus édulcorées. Elles n'ont pas besoin d'attirer l'attention sur le manque. La couleur de la Zone est liée au désir. Pour Anita, la seule fille de la bande de Magid et Pat, le voyou charmant dont elle rêve "sortirait d'entre deux tours peintes de toutes les couleurs pour planquer la grisaille du béton" (25-6). 
D'après Charef, "On ne se remet pas du béton. Il est partout présent, pesant, dans les gestes, dans la voix, dans le langage, jusqu'au fond des yeux, jusqu'au bout des ongles. Sur le bras il se transforme en trèfle à quatre fleurs tatoué en vert bouteille et dédié à sa mère, avec une rose" (58). Ce vert bouteille est loin d'être un faux-semblant. Il marque la chair comme les graffiti marquent les murs: "Ca chante pas, le béton, ça hurle au désepoir..." (59). Même l'esthétique en est empreinte. Contre le béton omniprésent, le tatouage serait "une amulette permanente, un bijou vivant qu'on ne peut pas enlever" (Michel Tournier). C'est à la fois puissant et douloureux. "Ne les laisse pas tomber, ils ont droit d'exister, eux aussi," nous chante Kacel dans "Banlieue." Charef ne trahit pas les siens: il dédie son roman à sa mère "même si elle ne sait pas lire" (5).

Le vert bouteille du Thé au harem n'a certainement rien à voir avec le vert de la nature, du thé à la mente, de la mer Méditerranée, ou de l'Islam. Mais c'est peut être la couleur qui convienne le mieux au Weltanschauung de Charef. Si l'esthétique ne semble pas échapper au béton, c'est qu'il y a pas de langage pauvre; il n'y a que le langage des pauvres. ${ }^{3}$ En s'écartant des couleurs adoucissantes, Charef rend la banlieue telle quelle est: dure, impitoyable, mais tout de même capable de donner lieu à une esthétique qui met les siens en valeur. Le roman et le film font ressortir le soutien infatiguable de Malika, à la fois aux siens et aux voisins, démontrant par là qu'il n'est pas nécessaire de "préférer sa fille à sa nièce, sa nièce à sa cousine, sa cousine à sa voisine" comme le soutiennent Le Pen et les apôtres de "La France aux Francais." Charef ne distingue pas entre Français de souche et immigrés maghrébins, dépassant par là certaines oppositions du roman "beur". C'est à Pat qu'on demande les papiers d'identité: "Je suis français, moi. Je suis dans mon pays. Tu me prends pour un Arabe, ou quoi?" (136). Chômeur, vadrouilleur, ami de basanés et noirs, Pat est un étranger chez lui. Même s'il est bien blanc. Qui s'assemble se ressemble. En effet, Charef renverse souvent les lieux communs (comme quand Majid et Pat "se font un métro"). A travers leur solidarité, les potes des la bande de Majid et Pat font face aux divisions ethniques et culturelles que la société française cherche à sauvegarder par les flics, les contremaîtres, et les petits chefs de l'ANPE ("On n'a rien pour vous, mon vieux!" 145). L'amitié devient presque une arme politique dans un roman et un film où la politique semble éscamontée. Car ces jeunes se soucient peu de la politique des pères, lesquels les ont pour la plupart désertés. En fait, ils s'en moquent des pères qui ont soit abandonné leurs mères en partant comme le père de Pat avec la voisine, soit interdit leurs fils de rentrer comme le père de Jean-Marc, soit poussé leurs filles au suicide en les enfermant à la maison quand elles sont enceintes, comme le père de Naima, soit affronté leurs fils avec matraques et gaz lacrymogènes quand ces derniers ont volé leurs réserves de vin. Quant au père de Majid, il "vogue sur une galère qui a paumé sa voile." (39) Majid ne peut rien attendre de cet homme qui était si dévoué à sa famille, car il a perdu la raison en tombant d'un toit. Les pères n'ont rien à leur léguer; ils doivent créer leurs propres racines: "Les racines n'étant pas ce que l'on traîne derrière soi, mais plutôt le but vers lequel l'on tend.".5

Quel est le but de ces personnages qui semblent être à la dérive, sans horizon d'avenir? 
A la fin, "las, dégoûté, fatigué" (182), Majid se rend à la police. Même l'amour pur n'est plus possible dans cette société. La femme que Magid aime finit par se prostituer pour gagner sa vie. Il s'agit de Chantal, la soeur de Pat. Que reste-t-il comme horizon d'espoir pour celui comme Magid qui refuse de devenir français quand les Français eux-mêmes sont au bout de la nuit? La dernière scène - à la fois du film et du roman - ouvre sur la mer, sur un moment de liberté où l'on voit les jeunes courir sur les vastes étendues des plages et sauter dans l'eau. Mais cet égarement dans la nature, celui réservé d'habitude aux bourgeois de Deauville, ne durera que quelques minutes à peine. Arrivera bien vite l'Estafette pour les arrêter, car un des jeunes a volé une BMW qui les a permis de venir ici, loin du béton étouffant. Ces quelques instants de plaisir ne sont qu'éphémères pour ces jeunes, délinquants ou non. Le spectateur ne peut s'empêcher de sourire avec eux en les voyant jouir de cette brève excursion hors de la banlieue. Mais le béton ne fera que se reserrer de plus fort: Majid, qui se laissera prendre par la police sans aucune résistance (ou aller en fait?) sera "béton." Comme Charef, son personnage ira en prison. A la fin, Majid s'endort dans cette Estafette qui roule "dans la nuit" (derniers mots, dernière couleur du roman). Mais pas avant d'avoir ramassé Pat. Car après s'être enfuit, Pat revient. Sa main levée en l'air pourrait être aussi bien celle du stop que celle du badge des années ' 85 "touche pas à mon pote." Cette dernière scène ne laisse pas le spectateur indifférent. D'après Jean-Michel Frodon, "On y subit une poussée de tendresse égale au volume de désespoir déplacé." Archimède cède la place à Archi Ahmed. L'amitié n'est peut-être pas un sujet si dérisoire pour ces jeunes dont l'horizon est la France multiculturelle d'aujourd'hui.

\section{Notes}

1 Le roman Le thé au harem d'Archi Ahmed de Mehdi Charef a paru chez Mercure de France en 1983. Le film "Le thé au harem d'Archimède" de Charef et Ray Costa-Gavras est sorti en 1985.

2 M. Lalonde, "Speak White" dans Défense et illustration de la langue québécoise suivie de prose et poèmes (Paris: Editions Seghers Laffont, 1980), p. 38.

${ }^{3}$ D'après André Pozner, "C'est frappant, si l'on parle d'un ouvrier, on ne dit pas qu'il a un langage de pauvre mais un langage pauvre, un vocabulaire pauvre (ce qui d'ailleurs est souvent faux)." Cité dans Anti-manuel de français de Claude Duneton et Jean-Pierre Pagliano (Paris: Seuil/Actuels, 1978/1980), p. 248.

${ }^{4}$ Voir à ce sujet Françoise Lévy, "Le Pen'Attention Inceste," L'Infini 13 (hiver 1986): 5861.

${ }^{5}$ Mehdi Charef cité dans "La percée des romanciers maghrébins et arabes en France" de Patrick Girard dans Jeune Afrique no. 1493-1494 (16 et 23 août 1989): 59.

6 Jean-Michel Frodon, "Mehdi Charef: naissance du cinéma 'beur'," Le Point no. 658 (29 avril 1985): 134. 